\title{
Human Immunodeficiency Virus Disease Death Rates* Among Women Aged 45-64 Years, by Race and Age Group - National Vital Statistics System, United States, 2000-2015
}

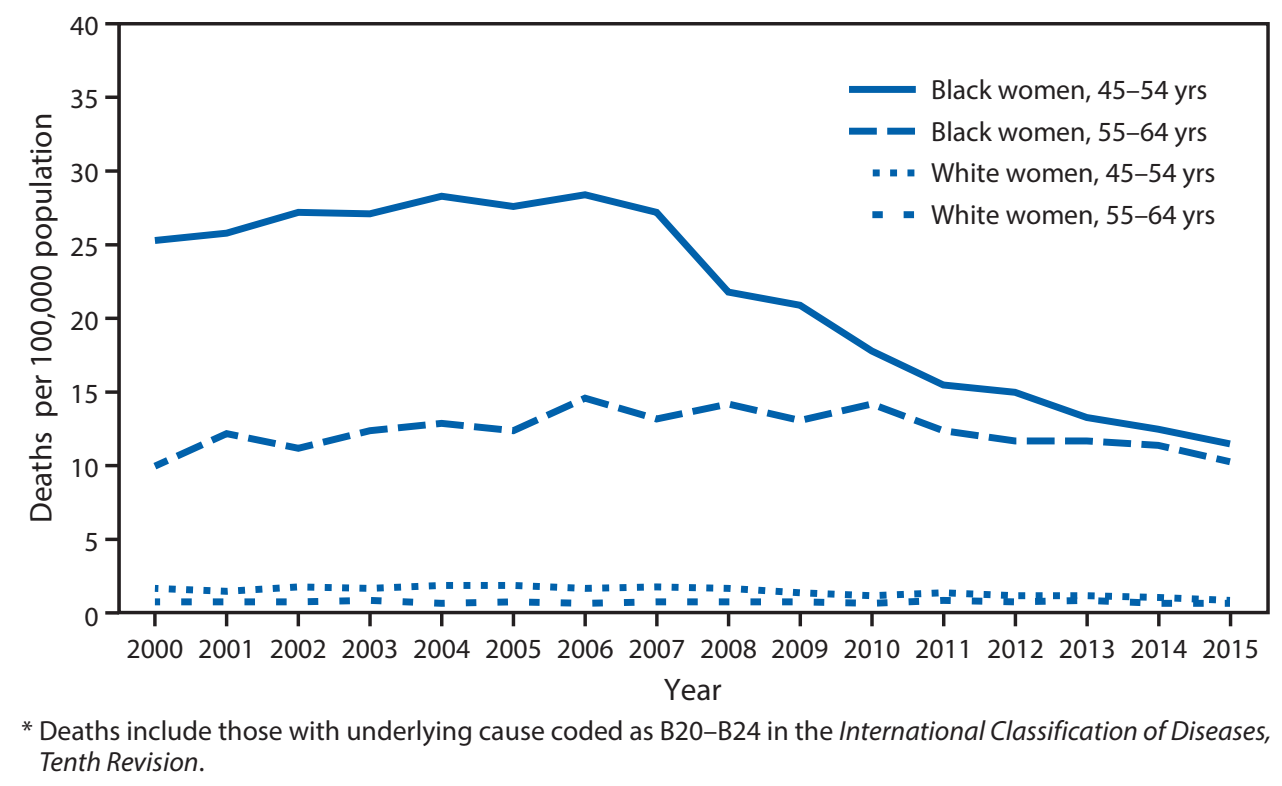

Among black women aged 45-54 years, the human immunodeficiency virus (HIV) disease death rate decreased $60 \%$ from 28.4 per 100,000 in 2006 to 11.5 in 2015. Among black women aged 55-64 years, the rate increased $42 \%$ from 10.0 in 2000 to 14.2 in 2008, before declining to 10.3 in 2015. Among white women aged $45-54$ years, the rate decreased $53 \%$ from 1.9 in 2005 to 0.9 in 2015. Among white women aged 55-64 years, the rate did not change, remaining at about 0.8 . Throughout the period, HIV disease death rates among black women were higher compared with rates among white women for both age groups.

Source: National Vital Statistics System. https://www.cdc.gov/nchs/data_access/vitalstatsonline.htm.

Reported by: Yelena Gorina, yag9@cdc.gov, 301-458-4241. 